\title{
The Role of Drug Exposure in Clinical Development: To What Extent Is Pharmacokinetic Assessment Needed in a Drug Development Programme?
}

\author{
Navin Goyal ${ }^{1}$
}

Published online: 27 May 2015

(C) Springer International Publishing Switzerland 2015

Drug development is a risky, lengthy and very expensive process. The centrepiece beneficiary of successful drug development rightfully is-and should always be-the patient. There is a perpetual effort to make pharmaceutical research and development simpler and faster in order to provide safe, efficacious and cost-effective drugs in a timely manner. As the candidate molecule progresses through various phases of clinical development (phases 1-3 and post-approval), data are generated that not only satisfy key regulatory requirements but also support internal investment risk assessments. There are some data (e.g. data on drug safety and tolerability) that are collected in all clinical trials of a molecule irrespective of whether the participants are healthy volunteers in phase 1 or patients in a phase 3 trial.

There have been various advances over the last decade to conduct fewer but better-designed studies and obviate duplicate, unwarranted, underpowered and inconclusive trials, e.g. adaptive study designs and model-based drug development approaches. These are resource efficient and ethical approaches to avoid unnecessary drug exposure in human subjects. Drug exposure or pharmacokinetic data are typically collected in almost all phases of drug development and often have a profound impact on the drug development programme in terms of cost and time. Importantly, pharmacokinetic data may be required by federal

This comment refers to the papers available at doi:10.1007/s40262015-0271-5 and doi:10.1007/s40262-015-0283-1.

\section{Navin Goyal}

navingoyal@gmail.com

1 Clinical Pharmacology, Modeling and Simulation, 709 Swedeland Road, King of Prussia, PA 19406, USA regulations in many scenarios, or strong justification may be needed if sponsors do not plan to collect/provide such data [1]. However, it should not be difficult to comprehend that whether mandated or not, pharmacokinetic data are critical to guide drug development and are often the sole end point of interest for certain specific studies. In some cases, a pharmacokinetic bridging study may potentially replace a large efficacy trial, e.g. in a paediatric population. The quantification of the exposure-response relationship assessment lies at the heart of any determination of the safety and effectiveness of new drug candidates. Hence, pharmacokinetic studies are critical for characterizing any intrinsic or extrinsic factors that could affect drug exposure.

The key studies conducted along the clinical journey of a candidate molecule are listed in Table 1, along with the criticality of drug exposure assessment in such studies. Each drug is unique, and these are not the only studies needed during development, nor are all of these studies always needed. Additionally, the sequence/timing of these studies is driven by drug safety, indication, benefit-risk assessment, the extent of 'at risk' investment a sponsor wants to make and the holistic perspective of existing and emerging data. It is also worth noting that not all studies are required to collect pharmacokinetic data to the same extent. While the majority of the listed studies employ a dense pharmacokinetic sampling strategy, confirmatory clinical and paediatric studies typically employ a modelbased approach (e.g. population analyses) to cut down pharmacokinetic sampling to the minimum essential level, or to avoid it completely, based on the question(s) to answer.

Gibbons et al. present summary data from multiple studies in this issue, providing a snapshot of exposure to 
Table 1 Key studies in clinical development that involve drug exposure assessment

\begin{tabular}{|c|c|c|}
\hline Study type & Phase $^{a}$ & Need for drug exposure assessment \\
\hline FTIH (single and multiple dose) & 1 & Essential \\
\hline DDI & $1-4$ & Essential \\
\hline Food effect & $1-2$ & Essential \\
\hline Relative BA/BE & $1-4$ & Essential \\
\hline Dose ranging & $1-2$ & $\begin{array}{l}\text { Preferred for characterization of exposure separation and/or exposure- } \\
\text { response }\end{array}$ \\
\hline QT-interval effects & $2-3$ & Highly recommended \\
\hline $\begin{array}{l}\text { Special populations such as patients with renal or hepatic } \\
\text { impairment }\end{array}$ & $2-4$ & Essential \\
\hline ADME & $1-3$ & Essential \\
\hline Confirmatory efficacy trials & $2 \mathrm{~b} / 3$ & $\begin{array}{l}\text { Preferred for understanding of demographic impact in a wider } \\
\text { population }\end{array}$ \\
\hline Paediatric population & $4^{\mathrm{b}}$ & Generally, pharmacokinetic data are essential \\
\hline
\end{tabular}

$A D M E$ absorption-distribution-metabolism-excretion, $B A$ bioavailability, $B E$ bioequivalence, $D D I$ drug-drug interaction, $F T I H$ first-time-inhumans

${ }^{a}$ This refers to when such studies are generally conducted, e.g. a healthy-volunteer DDI study may be conducted after an FTIH study on the basis of in vitro data and/or post-approval on the basis of emerging data from a wider population

b This is usually a post-approval commitment, unless the drug is primarily intended for a paediatric indication

enzalutamide and its key metabolites in healthy volunteers and patients requiring treatment for castration-resistant prostate cancer [2,3]. Exposure data from these key studies aid understanding of how different pieces come together and contribute towards drug approval and, importantly, any dose-related labelling recommendations. The studies discussed span from the first-time-in-humans (FTIH) study to drug-drug interaction (DDI) studies to the pivotal phase 3 efficacy trial. While these are not the only clinical studies conducted with enzalutamide, they have provided the majority of the data required to understand systemic drug exposure and dose selection.

For oncology drug candidates, FTIH trials may involve the target population rather than healthy volunteers, because of the perceived benefit-risk ratio and ethical considerations. Dose escalation occurs until safety signals emerge or an exposure threshold based on preclinical safety data is attained. Evaluation of single ascending doses followed by repeat or multiple-ascending-dose exploration provide the data for characterization of key pharmacokinetic parameters (e.g. the maximum concentration $\left[C_{\max }\right]$, area under the concentration-time curve [AUC] and elimination half-life $\left[t_{1 / 2}\right]$ ) and properties such as dose proportionality and drug accumulation. These data determine the dose and dosing regimen moving forward. Collection of biomarker data can aid exposure-response assessment and, with exposure separation across doses, enable choice of the optimal dose(s) for dose-finding or efficacy trials. For example, the choice of the $160 \mathrm{mg}$ dose in all studies of enzalutamide post-FTIH was predominantly supported by the analyses of drug exposure (pharmacokinetics), prostate-specific antigen reduction (pharmacodynamics) and safety data at different doses from the FTIH study, which appeared to enrol a relatively sufficient number of patients $(n=140)$. The pharmacokinetic-pharmacodynamic and safety rationale for the $160 \mathrm{mg}$ dose selection was not elaborated in these articles but can be found in another publication [4].

Regulatory guidance documents are available to help with appropriate design and conduct of the clinical pharmacology studies listed in Table 1. Incorporation of recommendations per such guidance documents ensures a certain level of scientific rigour and credibility of the data generated from such trials. While these studies have become somewhat standardized in nature, the drug pharmacokinetics and/or indication can bring about minor changes in the study design and size. Although crossover designs are generally recommended, parallel study designs are implemented with long-half-life drugs, e.g. enzalutamide. It is obvious that drug exposure measurement is essential in studies that evaluate food effects, renal or hepatic impairment, or drug metabolism. Reliable estimation of critical pharmacokinetic parameters requires adequate drug sampling, which, in turn, is drug dependent. Rich systemic sampling over more than 50 days was implemented in all phase 1 and 2 studies to adequately assess exposure to enzalutamide $\left(t_{1 / 2} \sim 4\right.$ days $)$ and its two major metabolites, $N$-desmethyl enzalutamide $\left(t_{1 / 2} \sim 8\right.$ days $)$ and the carboxylic acid metabolite $\left(t_{1 / 2} \sim 10\right.$ days). Nevertheless, instances do arise that require additional analyses for appropriate assessment of exposure beyond just summarizing the observed data. In silico modelling and simulation 
(M\&S) techniques have played an ever-increasing role in such circumstances, answering critical development questions and avoiding additional complex and lengthy studies or aiding efficient analyses of available data.

Assessment of DDI effects is an area where physiologically based pharmacokinetics (PBPK) and population $\mathrm{M} \& S$ have great utility. In vitro metabolism screens with recombinant cytochrome enzymes generally guide which specific DDI studies are conducted. On the basis of such experiments, a DDI study with the cytochrome P450 (CYP) 2C8 and CYP3A4 inhibitors gemfibrozil and itraconazole, respectively, was conducted with enzalutamide. The study involved a single enzalutamide dose administered in parallel arms with and without 3 weeks' dosing of the inhibitors and pharmacokinetic sampling for $>7$ weeks in a sufficient number of subjects. The major active metabolite, $N$-desmethyl-enzalutamide, with activity comparable to that of the parent compound and found at systemic levels similar to those of enzalutamide, was also measured. This study provides a good example of utilization of modelbased population analyses, the absence of which could lead to underestimation of the impact of DDIs [3]. The analyses recommended halving the $160 \mathrm{mg}$ dose of enzalutamide to $80 \mathrm{mg}$ when co-administering it with a CYP2C8 inhibitor to avoid the adverse events observed at exposures achieved with doses $>240 \mathrm{mg}$ in the FTIH study. The impact of enzalutamide on CYP2C8, CYP2C9, CYP2C19 and CYP3A4 substrates was demonstrated in an efficiently designed cocktail DDI study. This study design was in accordance with regulatory guidance and avoided multiple single DDI studies [5].

Some pharmacokinetic data may be collected in large phase 3 trials to understand drug behaviour in a wide range of demographics and, if possible, any exposure-response relationship. However, identifying any relevant relationship (pharmacokinetic and/or pharmacodynamic with the outcome) will depend on the range of the variable explored. To paraphrase, it may be impractical to characterize a dose-response relationship when only one dose is employed. Given that dose is a crude indicator of exposure, a more granular exposure variable, such as the AUC, $C_{\max }$ or $C_{\text {min }}$ (minimum concentration), may be evaluated instead of dose. Even so, this may not help establish-or may not be appropriate to establish-such a relationship. Consider the phase 3 AFFIRM trial in prostate cancer patients with one dose level - the $160 \mathrm{mg}$ dose. The study enrolled about 1199 subjects with 2:1 active-to-placebo randomization and documented $C_{\min }$ values of enzalutamide and its active metabolite on multiple occasions. The authors failed to establish any $C_{\min }$-survival relationship even with the huge sample size, which was not entirely surprising, given the low pharmacokinetic variability of the drug [2]. Nevertheless, these pharmacokinetic data, along with data collected from the various trials, were integrated with population modelling to evaluate the impact of characteristics such as age, weight, renal or hepatic impairment, and administration with meals on drug exposure. $M \& S$ can aid dose adjustment recommendations based on such modelbased analyses as long as adequate data are available.

Young scientists entering the drug development arena in academia, industry or regulatory agencies may understand the overall path of drug development but can fail to appreciate how a clinical dose is selected and carried forward across studies. Often, there may be a need to evaluate a dose different from what was approved when researchers are embarking on clinical studies in a new disease area, in a new patient population or in combination with other drugs. In such cases, the availability of all of the pharmacokinetic information across various studies, formulations and populations in a single comprehensive publication can be of immense value. Such summary publications highlight the role of adequately characterizing exposure to a drug and its key metabolite(s) in clinical development. It would be extremely difficult, if not impossible, for any drug development programme to move forward in the absence of pharmacokinetic data. These data can be used to develop drug concentration-disease-response models, which, in turn, can allow design of more efficient and fewer studies with possibly fewer drug exposure samples.

Compliance with ethical standards No funding was received for the preparation of this manuscript.

Conflict of interest Navin Goyal has no conflicts of interest to declare.

\section{References}

1. Code of Federal Regulations (CFR) Title 21, Office of the Federal Registrar, National Archives and Records Administration, USA; 2015.

2. Gibbons JA, et al. Clinical pharmacokinetic studies of enzalutamide. Clin Pharmacokinet. 2015. doi:10.1007/s40262-015-02715 .

3. Gibbons, JA, et al. Pharmacokinetic drug interaction studies with enzalutamide. Clin Pharmacokinet. 2015. doi:10.1007/s40262015-0283-1.

4. Scher HI, et al. Antitumour activity of MDV3100 in castrationresistant prostate cancer: a phase 1-2 study. Lancet. 2010; 375(9724):1437-46.

5. US Department of Health and Human Services. Guidance for industry, drug interaction studies-2012. http://www.fda.gov/ downloads/drugs/guidancecomplianceregulatoryinformation/ guidances/ucm292362.pdf. Accessed 1 May 2015. 